\title{
Social Media Addiction: A Systematic Review through Cognitive-Behavior Model of Pathological Use
}

\author{
Eiman Ahmed \\ Rutgers University \\ eiman.ahmed@rutgers.edu
}

\author{
Isaac Vaghefi \\ Pace University \\ sashrafvaghefi@pace.edu
}

\begin{abstract}
The proliferation of social media platforms such as Facebook, Twitter, and Instagram have revolutionized the way people communicate, consume, and share information. As a result, social media addiction, a type of behavioral addiction related to the compulsive use of social media and associated with adverse outcomes, has been discussed by scholars and practitioners alike. Despite the abundance of research published on social media addiction, this literature is fragmented, and there is no synthesis of the drivers and outcomes of this behavior. In this study, we use the cognitive-behavioral model of pathological use and conduct a systematic review of social media addiction literature from 20082019. Based on the review of 132 papers, we propose a framework that integrates prior findings. Our review reveals several avenues for future research on this increasingly prominent research topic.
\end{abstract}

\section{Introduction}

Before the emergence of the Internet, most information was circulated through major news outlets, and the majority of communication took place in person, through the phone, or using postal services. The Internet, which originated in 1991, transformed how information was shared and communication occurred by providing the public with an open platform to share and consume information without the involvement of any third-party organizations [1]. Social media platforms, by-products of the Internet, further perpetuated this revolution by supplying users with online communities to socialize and develop relationships.

Since its inception, social media platforms have garnered over three billion users worldwide and have grown exponentially in size [2]. According to a Pew Research Center report, social media users in the United States have increased by $1300 \%$ from 2005 to 2019 [3]. Social media usage has also risen dramatically, with a recent study showing that teenagers and young adults may spend up to 9 hours a day on social media [4]. Given these statistics, it is not surprising that many researchers have focused on exploring the possible effects of these platforms on users, particularly on the addictive nature of social media.

Social media addiction, the behavioral addiction related to the compulsive use of social media, has therefore been increasingly studied overtime. Similar to other known addictions, such as gambling, shopping, or sexual activity, it typically manifests through a set of symptoms, including salience, tolerance, mood modification, withdrawal, conflict, and relapse [5]. Although there is debate on the term addiction concerning technology use, social media addiction, also referred to as "compulsive," "excessive," "problematic," "maladaptive," or "pathological" social media use, has been studied extensively [6-10].

Studies have used a wide variety of theories, frameworks, and methodologies, to shed light on the possible determinants and outcomes of social media addiction. In terms of antecedents, studies have found that certain mental disorders such as anxiety, depression, and ADHD are all positively correlated to social media addiction [11]. They have also found that users with certain personality types, particularly users who are more extroverted, open to new experiences, agreeable, and neurotic, are at higher risk than others for developing an addiction to social media $[8,12]$. In terms of effects, studies have shown that social media addiction could lead to stress, relationship problems, and life dissatisfaction, amongst other complications [13-15].

Although research on the drivers and outcomes of social media addiction is accumulating and provides significant theoretical and practical insights, these efforts sometimes provide contradictory findings. For example, whereas some studies have shown that depression is a predictor of social media addiction [11, 12], others have found that depression is an outcome of addiction $[10,16]$. Similarly, whereas some findings suggest that having an extroverted personality is positively related to social media addiction [15], others have found that having an extroverted personality is, in fact, negatively associated with addiction [17].

These disparities in existing research make it challenging to consolidate knowledge and reach a consensus about the true nature of social media addiction and the network of factors related to it. To 
address this issue and advance the current state of research, we aim to address two research questions in this study: 1) What are the factors underlying social media addiction? 2) What are the outcomes of social media addiction?

To answer these questions, we conduct a systematic literature review on social media addiction from 2008 to 2019. We use the cognitive-behavioral model of pathological Internet use [18] as a sensitizing tool to integrate the findings of 132 relevant papers. Based on our review, we provide avenues for future research on this topic.

\section{Cognitive-Behavioral Model of Pathological Use}

To conduct our review, we draw upon the cognitivebehavioral model (CBM). Proposed by Davis [18], $\mathrm{CBM}$ is one of the major frameworks used to explain factors related to Internet-based addictions. The model states that maladaptive thoughts in congruence with social isolation are the primary cause of pathological Internet use (PIU).

CBM relies on the notion of two types of antecedent factors, distal and proximal, both contributing to PIU. Proximal factors lie at the end of the etiology chain and are sufficient for PIU to occur, while distal factors lie at the beginning of the chain and are insufficient by themselves.

In CBM, psychopathological symptoms such as depression or anxiety and the Internet or Internet-related activities are distal necessary causes; they must be present for PIU to occur. Situational cues such as the sound of a keyboard are also distal factors; however, they are not coined necessary as they only lead to conditioned responses in some individuals.

In contrast, maladaptive cognitions and social isolation or lack of social support are proximal causes of PIU. There are two types of maladaptive thoughts in CBM: about oneself and about the world. Maladaptive cognitions about oneself typically result from conditions such as self-doubt, low self-efficacy, and low selfesteem and are related to using the Internet for selfappraisal. Maladaptive cognitions about the world, on the other hand, pertain to using the Internet as a substitute for the "real world."

According to CBM, there are also two types of PIU, specific and generalized. Specific PIU is associated with overusing particular Internet functions to satisfy preexisting disorders, such as gambling, online. On the contrary, generalized PIU is related to spending excessive time on the Internet without a particular purpose. In CBM, maladaptive cognitions on their own lead to specific PIU and maladaptive cognitions in unison with social isolation cause generalized PIU.

We use CBM to interpret the findings of our review for several reasons. First, the model has received attention from various scholars, many of whom have empirically evaluated the framework's validity $[19,20]$. Second, the theory has a broad focus. It explores how disorders such as depression and anxiety lead to maladaptive thinking and, in turn, compulsive behavior and unfavorable outcomes. CBM's broad focus and its distinction of generalized PIU as an Internet-specific phenomenon allow the model to be malleable enough to be applied to several Internet-related disorders, such as problematic video-gaming and mobile phone dependence [21], or in our case, social media addiction.

\section{Methodology}

To conduct our review, we searched the keywords "social media" and "social networking" in combination with "addiction" on the Web of Science's Core Collection (of journals). We limited our search to journal articles published from 2008 to 2019 and excluded proceeding papers, book chapters, and editorials creating an initial database of 1522 papers.

From this collection, we removed publications, not in English, and publications from journals outside of the

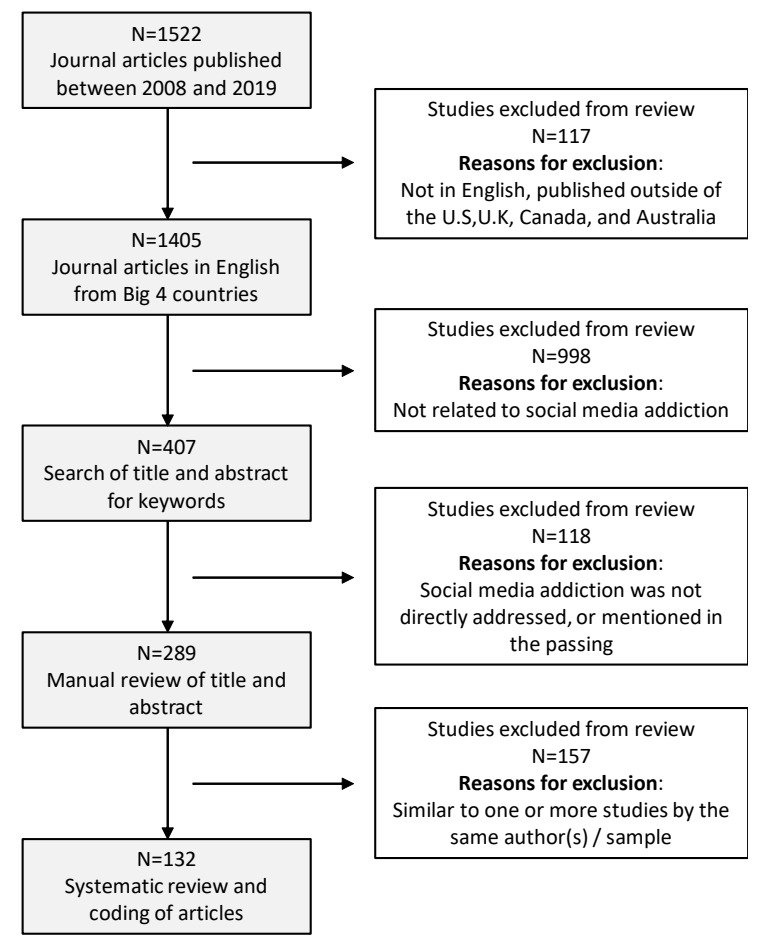

Figure 1. Paper Selection Process

United States, the United Kingdom, Canada, and 
Australia. We discarded a total of 117 papers in this step for a remainder of 1405 .

Using a similar approach to Beaudry et al. [22], we further removed papers whose titles and abstracts did not discuss social media addiction. This action resulted in the dismissal of an additional 998 papers for a new remainder of 407.

After, using systematic review principles discussed by Petticrew and Roberts [23], we read relevant papers' abstracts to address their applicability to our topic and removed articles that studied other technology addictions such as online gaming addiction and Internet addiction. We discarded an additional 118 papers in this step, for a remainder of 289.

After that, we excluded papers with the same participants (i.e., several publications on the closely related topic by the same authors). This procedure resulted in the removal of 157 papers for a final remainder of 132 (see Figure 1).

We then read each article successively, recording information regarding methodology, theoretical background, and social media platform studied. We also documented each study's population, sample size, highest level of analysis, findings, independent variables, and dependent variables. Lastly, we used $\mathrm{CBM}$ as a sensitizing tool to consolidate our findings and provide an overview of social media addiction literature. We used the information we cataloged in our database to summarize past findings, extend CBM, identify strengths in prior research, and offer suggestions for future research.

\section{Findings}

\subsection{Descriptive Statistics}

Various theoretical models have been used to study social media addiction. Our review revealed a total of 93 different theories, from a variety of disciplines, with some theories looking into the influence of personality traits and some others investigating the role of IT artifact on social media addiction. From these theories, the most used theories to study social media addiction were the uses and gratification theory (UGT) (15\%), CBM (14\%), and the five-factor model of personality (FFM) $(5 \%)[18,24,25]$.

Besides, many social media platforms have been focused on in prior studies of social media addiction. In our review, we found that while the majority of papers (33\%) investigated Facebook, 20\% explored other platforms such as YouTube and Twitter [24, 26].

\footnotetext{
${ }^{1}$ Note that framing the factors as direct antecedent or a moderator of social media addiction was done theoretically, and according to the original CBM. Other complex direct,
}

Nevertheless, we discovered that the majority of papers $(55 \%)$ preferred to study social media generically and as a whole, rather than specifying an individual platform [12, 27].

Populations from several countries were also examined in relation to addiction. Specifically, we found that studies' research data was collected from 35 distinct countries. Most papers (23\%) explored addiction in the United States, while many papers also looked into countries such as China (11\%), Italy (6\%), and the United Kingdom (4\%) [6, 27-29].

In addition, different populations of users were also researched. Most papers in our review (68\%) studied social media addiction in the context of students [24, $30]$. Meanwhile, $26 \%$ of papers explored addiction in adolescents, young adults, adults, and other populations, and $20 \%$ examined social media users in general $[8,27$, 31]. The sample sizes in these studies ranged from a few hundred to several thousand. Yet, the majority of papers in our review (67\%) investigated samples of less than $500[12,16]$. Besides, $24 \%$ looked into samples of 500 to $1000[15,32]$, and a few papers $(3 \%)$ examined populations of 5000 or above [33, 34].

In regard to gender characteristics and the likelihood of social media addiction, our review overall suggested that, as supported by $9 \%$ of papers, women and younger populations are at a higher risk of developing social media addiction compared to the general population [33].

\subsection{Results of Systematic Review}

Overall, our systematic review showed that CBM is a suitable theoretical model for capturing the findings of social media addiction literature. We found that prior findings can be suitably mapped onto the elements of $\mathrm{CBM}$ to varying degrees; some elements, such as psychopathology, received more attention than others, such as situational cues. Based on our review, we argue that $\mathrm{CBM}$ can be extended. While the original model has shown significant theoretical value and validity, research progress and accumulating empirical evidence call for an update of the model that includes additional elements, such as individual needs and motivations, personality traits, socio-environmental factors, and addiction outcomes (see Figure $2^{1}$ ). We provide details about each element of our review in the next section.

\subsubsection{Distal factors}

moderating or mediating effects are likely and sometimes supported by existing findings. 
Social media: According to CBM, IT artifact (the online environment that users interact with) plays a significant role in the development of PIU. In our context, the IT artifact required for social media addiction to occur is the social media platform or specific characteristics or features of social media that could influence addiction. Indeed, 24 papers discussed the role of social media on addiction in our review.

The majority of papers in our review (12/24) explored the impact of usage duration on addiction. Usage duration can be defined as the amount of time users spend on social media. Although spending excessive time on Internet applications does not necessarily constitute an addiction, scholars have consistently indicated that time online is a crucial determinant of technology addictions [17]. Papers in our review showed support for this relationship. For instance, in a study by Ndasauka, et al. [17], researchers found that the more time users spent on Twitter, the more likely they were to become addicted. Similar findings were reported in the context of Facebook [26] and general social media use [33]. Four papers (out of 24) also researched the effect of frequency or intensity of use on social media addiction. Whereas the frequency of use can be defined as the repeated use of social media throughout the day, the intensity of use can be explained as the emotional use of social media. These papers found that both factors were positively associated with addiction [35-37].

At a more granular level, a handful of papers from our review (5/24) studied the impact of specific features on social media addiction. Social media are composed of several features that allow users to interact with one another in many ways. When examining social media environments, thus, it is also essential to take into account the features that make up those environments. Papers that investigated the impact of specific features on addiction found that social media notifications, status updates, likes, friend counts, and games, all positively contributed to social media addiction [24, 32, 38, 39].

Finally, the findings of Abbasi [40]'s study revealed that having several concurrent social media accounts increased the likelihood of developing an addiction to social media, while van Rooij, et al. [41] showed that having a Twitter account also puts users at a higher risk of addiction to other social media.

Individual Needs/Motivations: Existing research findings suggest that receiving gratification for specific needs or motives could lead to pathological use [42]. Accordingly, we identified a significant body of research that focused on the individual needs underlying the development of addiction. With this focus, we found 20 papers, addressing various types of factors related to specific needs or particular motivations that can be fulfilled via social media use.

The most common category was to use social media in order to develop and manage social relationships. Social media provides users with various means to interact and communicate with other people. Based on our review, 11 papers (out of 20) addressed various social or relational needs. For instance, Sural, et al. [43] and Masur, et al. [44] showed that users driven by the desire to form new relationships on social media were more prone to addiction development. Similarly, Sural, et al. [43] and Alzougool [31] revealed that users that used social media to maintain existing relationships were also susceptible to social media addiction.

Many papers in our review (7/20) explored the role of entertainment-related motives on addiction. Social media presents several avenues for entertainment, from content sharing to gaming. Our review revealed that users who use social media to satisfy entertainment needs are at risk of developing an addiction. For example, in a study by Balakrishnan and Griffiths [24], scholars found that using YouTube with an inclination

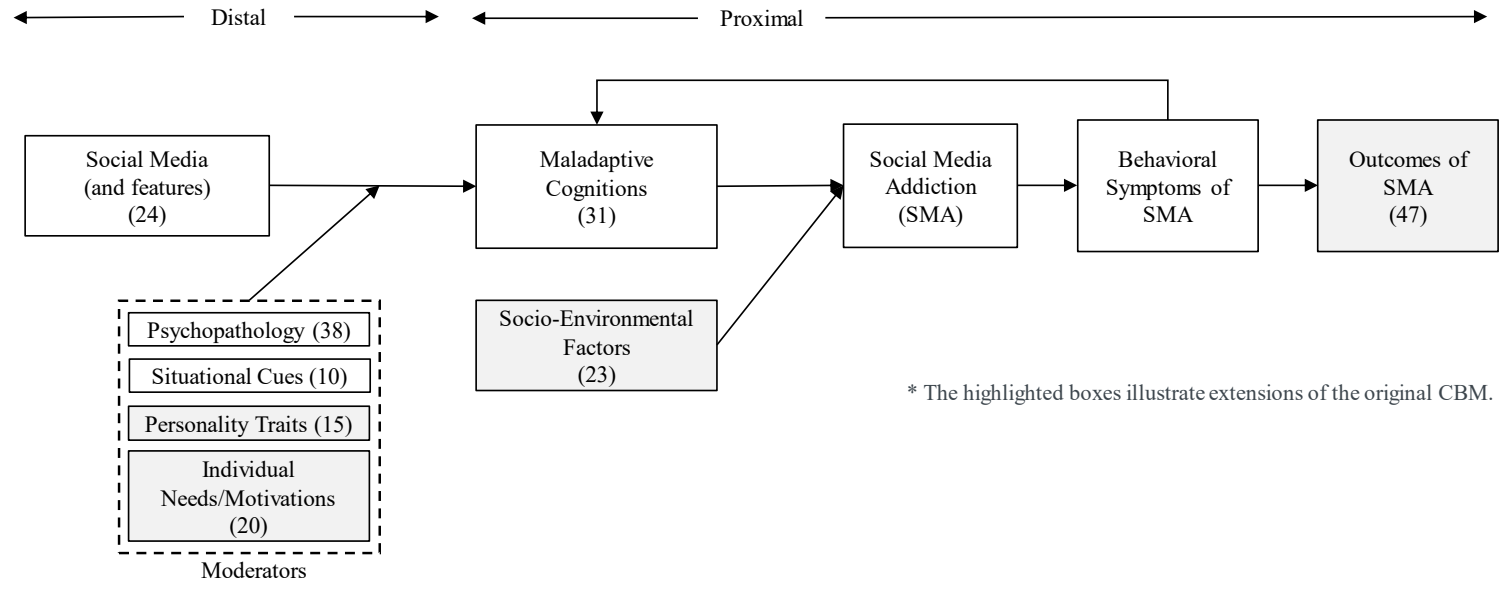

Figure 2. A Revised Cognitive-Behavioral Model of Social Media Addiction 
to view content made users susceptible to YouTube addiction.

In addition, we found five papers (out of 20) that examined socialization needs. Intended by design, social media provides many alternatives to satisfy social needs, such as private messaging, groups, and comments. Similar to entertainment needs, we found that users who receive gratification for social needs from use are more likely to develop an addiction [31].

In addition to these findings, we also found that individual needs and motivations could moderate the relationship between social media and maladaptive cognitions or addiction. For instance, in a study by Koc and Gulyagci [26], it was found that using Facebook specifically for social purposes made users more vulnerable to social media addiction. This study corroborates that using social media to satisfy specific needs could moderate the relationship between social media and maladaptive cognitions.

Psychopathology: CBM views psychopathology as the predisposed vulnerability that results in PIU. A large percentage of papers in our review (29\%) provided some support for the role of psychopathology (and its related factors) on social media addiction.

Most of these papers (26/38) explored the impact of depression and anxiety on addiction. Of the papers that studied anxiety (12/38), five looked into social anxiety, while four examined attachment anxiety, and three others investigated general anxiety. Prior research indicates that individuals suffering from mental disorders and, in particular, psychological and emotional disorders, are more vulnerable to addiction [11]. Our review underlines these findings in the context of social media addiction and reveals that these factors have a significant negative influence on addiction [16].

Besides, other papers in our review (3/38) paid research attention to the impact of attentiondeficit/hyperactivity disorder or ADHD on addiction. Research suggests that individuals with ADHD are more prone than others to developing behavioral addictions since they have impaired impulse control [6]. Indeed, ADHD was found to positively influence social media addiction in our review [6].

A few papers researched comorbidity of other addiction types on social media addiction. Ceballos, et al. [45] examined the role of binge drinking on addiction and found that the quantity and frequency of alcohol use were linked to higher addiction levels in college students. Moreau, et al. [46] looked into borderline traits and revealed that they were positively associated with Facebook addiction. Furthermore, Andreassen, et al. [11] looked into the impact of obsessive-compulsive disorder on addiction and found that it had similar effects.
Similar to individual needs and motivations, we found that these actors could also moderate the relationship between social media and maladaptive cognitions or addiction. For example, Chung, et al. [47] concluded that the existence of psychopathological conditions during social media usage increases the likelihood of social media addiction and the emergence of addiction symptoms. This study confirms that psychopathological factors could also moderate the relationship between social media and maladaptive cognitions.

Situational Cues: Situational cues are secondary reinforcers that lead to conditioned compulsive responses. According to Davis [18], "stimuli such as the sound of a computer connecting with an online service, the tactile sensation of typing on a keyboard, and even the odor of one's office or primary place of using the Internet" are considered situational cues. Compared to other elements of CBM, fewer papers in our review (10) discussed factors related to situational cues and social media addiction.

From these papers, three (out of 10) focused on the use of other potentially addicting technologies, such as the Internet or mobile phones, to see whether concurrent use of these technologies can speed up the development of social media addiction. The findings of these papers show that the use of the Internet or mobile phone intertwines with social media in users' memory so that anytime these other technologies are accessed, social media use is prompted, and the likelihood of developing social media addiction increases $[25,32]$.

Besides, a few studies (2/10) looked at the environment around use and investigated whether using social media during specific times influences addiction. The papers found that habitual use of social media on the weekends or at night (right before bed), compared to typical use during the week or daytime, is associated with higher addiction levels [25, 48]. Lastly, one study (out of 10) found support for alcohol consumption being positively correlated with social media [45]. In all cases, these studies' findings suggest that when individuals frequently observe or are at the reach of various skills related to social media use, they have a higher likelihood of developing social media addiction.

Similar to psychopathology, we found that situational cues could moderate the relationship between social media and maladaptive cognitions. For instance, in a paper by Li, et al. [48], it was found that intense social media use during the weekend could positively moderate the effect of the direct antecedents and put users at higher risk of developing addiction than non-weekend users. Therefore, in line with CBM, we consider the situational cues related to social media use as potential moderators of the relationship between social media and maladaptive cognitions. 
Personality Traits: While not initially considered in CBM, we found that a significant body of research has been done to establish the association between personality traits and social media addiction.

These studies (15) typically used the five-factor model of personality to examine the association between higher-order traits (i.e., openness to experience, conscientiousness, extroversion, agreeableness, and neuroticism) and addiction [30]. While eleven papers (out of 15) considered at least one of the five factors in their examination, others $(4 / 15)$ examined other traits that were deemed relevant. Despite this, the findings of these papers have been inconsistent except for the effect of conscientiousness $(8 / 15)$, which is shown to help reduce addiction $[12,49]$. The evidence for the effect of other traits has been mixed and inconclusive, pointing to both positive and negative directions. While some papers show that extroversion, neuroticism, openness, and agreeableness are positively associated to social media addiction, others show that they are, in fact, negatively linked instead. In the case of neuroticism, while many papers $(4 / 15)[8,12]$ revealed that the trait was positively associated with addiction, a paper by Tang, et al. [49] showed that neuroticism had an indirect relationship with social media addiction. Similarly, whereas many papers $(5 / 15)[8,30]$ found a direct relationship between extroversion and addiction, some (2/15) showed that this trait was actually negatively related to addiction [12]. Next, although a few articles $(2 / 15)[30,50]$ found that openness decreased the likelihood of social media addiction, Dalvi-Esfahani, et al. [12] and Caci, et al. [25] revealed the opposite correspondence. Lastly, while some studies (3/15) [32, 49] showed that being agreeable increased the risk of developing an addiction to social media, a study by Dalvi-Esfahani, et al. [12] noted the reverse effect.

The role of other (less studied) personality traits was also investigated in the context of social media addiction. In specific, three papers in our review (out of 15) researched the impact of narcissism on social media addiction. These papers underlined the findings of past studies suggesting that the trait was linked to higher IT and social media addiction levels [12]. On the contrary, a study by Iranmanesh, et al. [51] explored the role of shyness and showed that it too increased the risk of addiction. Lastly, Dalvi-Esfahani, et al. [12] found that empathy was negatively related to addiction.

Similar to the elements mentioned above, we found that personality traits could also moderate the relationship between social media and maladaptive cognitions. Brailovskaia and Margraf [16], for instance, found that narcissism exacerbated the impact of intense Facebook use on social media addiction. Thus, we regard personality traits as possible moderators of the relationship between maladaptive cognitions and social media.

\subsubsection{Proximal factors}

Maladaptive Cognitions: In CBM, maladaptive thoughts stem from negative views of either the world or oneself and cause users to become addicted to social media. In our review, we found that maladaptive cognitions received significant attention in prior social media research since near a quarter of papers (31) in our sample provided some theoretical insight on factors related to negative thoughts about the self or the world.

In terms of the self, we found that most papers in our review (11/31) examined the link between self-esteem (i.e., individuals' perception of self-ability) and social media use or addiction. The findings overall indicate that lower self-esteem encourages further use (an indirect effect) and promotes addiction $[8,34]$. Research shows that users with low self-esteem often turn to social media to improve their perception of themselves [34]. Accordingly, social media's use as a response to some negative cognitions also appeared to provide grounds for social media addiction. For instance, Foroughi, et al. [52] found that users who used social media to gain recognition or gratification from others, be that due to low self-esteem or other reasons, were more prone to develop an addiction to social media [53]. These findings were replicated by several papers in our review $(6 / 31)$.

In terms of the negative thoughts about the world, four papers (out of 31) in our review looked into the role of escapism on addiction. Escapism can broadly be defined as the need to get away from the "real world." In the same way that users with maladaptive thoughts about themselves use social media to feel better, users with maladaptive thoughts about the world use social media to do the same. Similar to the effect of the previously mentioned variables in this section, findings from our review suggest that escapism also increases the odds of developing social media addiction $[29,44]$.

Socio-Environmental Factors: While CBM only considers social isolation or lack of social support as proximal environmental factors that exacerbate generalized PIU, based on our review results, we broadened the definition of this element to include factors related to social environment as well as personal struggles (hence, labeled as socio-environmental factors). With this expanded definition, we found 23 papers addressing related variables.

Similar to maladaptive cognitions about the world, these factors often relate to feeling unfulfilled in the "real world." Many papers in our review (8/23) researched the impact of loneliness on addiction. Loneliness is a subjective feeling that may result from 
social isolation or false beliefs. We found support from several papers indicating that users who are lonelier, are more susceptible to developing social media addiction than others [12, 51]. Many papers also studied how the two extremes of isolation (initially mentioned in CBM) can be detrimental toward social media use. Results from our review show that while a lack of in-person social support can contribute to addiction, the surplus of online social support provides similar effects $[17,49]$.

Besides factors directly related to the social environment surrounding individuals, some papers in our review (6/23) looked into the role of other aspects of everyday life on addiction. Dalvi-Esfahani, et al. [12], for example, found that life satisfaction was indirectly linked to social media addiction.

\subsubsection{Outcomes}

While initially not considered by CBM, our review of the nomological network of social media addiction sheds light on various potential outcomes of these behaviors, which we included in the revised framework. Our review revealed 47 papers that addressed the outcomes that resulted from social media addiction.

From these papers, a majority (17/47) considered the adverse outcomes of addiction on individuals' mental and physical well-being. Brailovskaia and Margraf [16] found that social media addiction challenged users' overall well-being by increasing stress symptoms. Similarly, Atroszko, et al. [15] revealed that social media addiction negatively impacted physical health by reducing sleep quality. Lastly, Benson, et al. [28] showed that addiction also reduced users' ability to perform well physically (in the context of sports or other related physical activity).

In line with mental well-being, many papers in our review (14/47) specifically analyzed the effects of social media addiction on psychopathology. Whereas some studies looked into psychological well-being as a whole [54], others looked into distinct psychopathologies such as depression and anxiety, as outcomes of addiction. Eight studies (out of 47) found support for the positive association of addiction with depression [16, 52]. One study by Foroughi, et al. [52] provided support for the direct relationship between addiction and social anxiety. Additionally, some studies in our review found that addiction to social media increased the likelihood of developing other technology addictions such as Internet gaming disorder and mobile phone addiction $[55,56]$.

Although social media provides users with avenues to fulfill socialization needs, several papers in our review (11/47) showed that excessive social media use and addiction might actually increase social burdens. For instance, Elphinston and Noller [13] showed that addicted users were more dissatisfied with their social relationships than were their counterparts. In a separate study, Caci, et al. [25] found that addiction to social media reduced the quality of social relationships.

Finally, many papers in our review (8/47) also explored the impact of addiction on users' professional lives. They found that social media addiction caused problems in both school and work settings. For instance, studies found that addicted social media users had increased difficulty in accomplishing work-related tasks [57] and had a lower GPA [58].

\section{Discussion}

The goal of our study was to provide a systematic review of the literature on social media addiction using CBM as a sensitizing tool to summarize the findings. Our review showed that social media addiction research covers a range of factors, from psychopathology to maladaptive cognitions. It also covered both determinants and outcomes of this addiction. In terms of antecedents, our review revealed that depression, low self-esteem, usage duration, entertainment motives, and neuroticism, to name a few, could positively predict social media addiction. Additionally, regarding outcomes of addiction, our paper showed that social media addiction could lead to depression, lower academic performance, deteriorated well-being, and lower quality of social relationships.

From this review, we conclude that social media addiction shares similarities with other types of technology addictions, such as online gaming addiction. Furthermore, our review revealed that comorbid addictions could augment each other's symptoms. For instance, Pontes [56] found that whereas Internet gaming addiction (IGD) was positively associated with social media addiction, social media addiction was also positively associated with IGD.

Additionally, our study demonstrated the potential bidirectionality of several factors related to social media addiction. Specifically, it showed that some factors that predict addiction (particularly those related to psychopathology and maladaptive cognitions, such as depression and anxiety) also might result from social media addiction (see the feedback arrow in Figure 2).

Individuals with psychopathological symptoms often turn to the Internet to find relief for their symptoms. Our review showed that if users were to find relief from Internet applications, that relief might be temporary, and Internet applications, including social media, could actually exacerbate existing psychopathological conditions of users. In the same manner, our review revealed that although individuals may use social media for relationship maintenance or socialization purposes, social media might in fact do more harm than good, since our review indicated that 
addiction is associated with relationship dissatisfaction and lower quality of social relationships.

Our study makes several contributions to existing research that uses CBM. First, it challenges the idea that, as proposed by CBM, technology addictions stem from maladaptive thinking. For example, it revealed that although many factors related to maladaptive cognitions are associated with social media addiction (i.e., low selfesteem and low self-efficacy), other factors-irrelevant to maladaptive cognitions such as extroversion and narcissism can trigger social media addiction. Thus, it is unclear whether social media addiction is truly a byproduct of maladaptive thinking or other elements.

Furthermore, although our study showed that CBM is an appropriate model to encompass the findings of social media addiction literature, it also revealed that modifications might be necessary to provide a complete picture of the etiology of social media addiction. Additionally, our study highlighted the need for supplemental research concerning various mixed or contradictory findings from existing social media addiction literature. Our review showed that some variables, such as extroversion, were somehow both positively and negatively related to social media addiction. Thus, the relationships between these findings and social media addiction appear to be mixed, which calls for additional research.

Lastly, our study illustrated the need for further research regarding some aspects of CBM and social media addiction. Based on our review, we found that there is a paucity of research investigating the impact of the IT artifact and its characteristics on addiction. Given the emphasis CBM put on the role of the Internet in the development of PIU, further research is required to understand the role of social media and its features on addiction.

Furthermore, additional research with a focus on general populations is needed. In our study, we found that most papers investigated social media addiction among students and/or younger populations. These samples, although typically representative, cannot provide a complete picture of social media addiction (e.g., how older adults may develop an addiction). In order to gain a more accurate understanding, further research utilizing other populations is required.

Overall, our findings revealed that, although existing research on social media addiction provides significant theoretical and empirical insights, there is a need for additional research on underexplored domains.

\section{Conclusion}

The purpose of our study was to provide an overview of social media addiction literature by conducting a systematic review of papers that met the inclusion/exclusion criteria (132). Using CBM to categorize findings, our review revealed the current state of research on the antecedents and outcomes of social media addiction. Our study complements prior studies that have evaluated the validity of CBM with relevance to technology addictions; yet, it provides an update of the model, based on the findings of this systematic review.

\section{References}

[1] I. Vaghefi and L. Lapointe, "Can IT hurt productivity? An investigation of IT addiction," presented at the Proceedings of the 34th international conference on information systems, Milan, Italy, 2013. [2] P. G. Cooper, "Social Media," in Salem Press Encyclopedia, ed, 2019.

[3] Pew Research Center. "Social Media Fact Sheet." https://www.pewresearch.org/internet/fact-sheet/socialmedia/ (accessed 23 Jun, 2020).

[4] I. Vaghefi and H. Qahri-Saremi, "Personality Predictors of IT Addiction," in Proceedings of the 51st

Hawaii International Conference on System Sciences, 2018 ,

[5] I. Vaghefi, L. Lapointe, and C. BoudreauPinsonneault, "A typology of user liability to IT addiction," Information Systems Journal, 27, 2, 2017, pp. $125-169$

[6] M. Settanni, D. Marengo, M. A. Fabris, and C. Longobardi, "The interplay between ADHD symptoms and time perspective in addictive social media use: A study on adolescent Facebook users," Children and Youth Services Review, 89, 2018, pp. 165-170

[7] V. Apaolaza, P. Hartmann, C. D'Souza, and A. Gilsanz, "Mindfulness, Compulsive Mobile Social Media Use, and Derived Stress: The Mediating Roles of Self-Esteem and Social Anxiety," Cyberpsychology Behavior and Social Networking, 22, 6, 2019, pp. 388-396

[8] S. S. Ho, M. O. Lwin, and E. W. J. Lee, "Till logout do us part? Comparison of factors predicting excessive social network sites use and addiction between Singaporean adolescents and adults," Computers in Human Behavior, 75, 2017, pp. 632-642 [9] X. L. Hou, H. Z. Wang, C. Guo, J. Gaskin, D. H. Rost, and J. L. Wang, "Psychological resilience can help combat the effect of stress on problematic social networking site usage," Personality and Individual Differences, 109, 2017, pp. 61-66

[10] H. G. Holmgren and S. M. Coyne, "Can't stop scrolling!: pathological use of social networking sites in emerging adulthood," Addiction Research \& Theory, 25, 5, 2017, pp. 375-382

[11] C. S. Andreassen et al., "The Relationship Between Addictive Use of Social Media and Video 
Games and Symptoms of Psychiatric Disorders: A Large-Scale Cross-Sectional Study," Psychology of Addictive Behaviors, 30, 2, 2016, pp. 252-262 [12] M. Dalvi-Esfahani, A. Niknafs, D. J. Kuss, M. Nilashi, and S. Afrough, "Social media addiction: Applying the DEMATEL approach," Telematics and Informatics, 43, 2019,

[13] R. A. Elphinston and P. Noller, "Time to Face It! Facebook Intrusion and the Implications for Romantic Jealousy and Relationship Satisfaction,"

Cyberpsychology Behavior and Social Networking, 14, 11, 2011, pp. 631-635

[14] O. Turel and I. Vaghefi, "Social media detox: Relapse predictors," Psychiatry research, 2019, p. 112488

[15] P. A. Atroszko, J. M. Balcerowska, P.

Bereznowski, A. Biernatowska, S. Pallesen, and C. S. Andreassen, "Facebook addiction among Polish undergraduate students: Validity of measurement and relationship with personality and well-being," Computers in Human Behavior, 85, 2018, pp. 329-338

[16] J. Brailovskaia and J. Margraf, "Facebook Addiction Disorder (FAD) among German students-A longitudinal approach," Plos One, 12, 12, 2017, [17] Y. Ndasauka et al., "Excessive use of Twitter among college students in the UK: Validation of the Microblog Excessive Use Scale and relationship to social interaction and loneliness," Computers in Human Behavior, 55, 2016, pp. 963-971

[18] R. A. Davis, "A cognitive-behavioral model of pathological Internet use," Computers in Human

Behavior, 17, 2, 2001, pp. 187-195

[19] R. A. Davis, G. L. Flett, and A. Besser, "Validation of a new scale for measuring problematic internet use: implications for pre-employment screening," (in eng), Cyberpsychol Behav, 5, 4, 2002, pp. 331-45

[20] S. S. J. Lin and C.-C. Tsai, "Sensation seeking and internet dependence of Taiwanese high school adolescents," Computers in Human Behavior, 18, 4, 2002, pp. 411-426

[21] C. J. Forrest, D. L. King, and P. H. Delfabbro, "The measurement of maladaptive cognitions underlying problematic video-game playing among adults," Computers in Human Behavior, 55, Part A, 2016, pp. 399-405

[22] A. Beaudry, I. Vaghefi, L. Lapointe, and F. Bagayogo, "Impacts of IT User Behavior:

Observations Through A New Lens," Communications of the Association for Information Systems, 46, 1, 2020, pp. 331-364

[23] M. Petticrew and H. Roberts, Systematic Reviews in the Social Sciences: A Practical Guide (Systematic Reviews in the Social Sciences: A Practical Guide).
Oxford: Blackwell Science Publ (in English), 2006, pp. $1-336$.

[24] J. Balakrishnan and M. D. Griffiths, "Social media addiction: What is the role of content in YouTube?,"

Journal of Behavioral Addictions, 6, 3, 2017, pp. 364377

[25] B. Caci, M. Cardaci, F. Scrima, and M. E. Tabacchi, "The Dimensions of Facebook Addiction as Measured by Facebook Addiction Italian Questionnaire and Their Relationships with Individual Differences," Cyberpsychology Behavior and Social Networking, 20, 4, 2017, pp. 251-258

[26] M. Koc and S. Gulyagci, "Facebook Addiction Among Turkish College Students: The Role of Psychological Health, Demographic, and Usage Characteristics," Cyberpsychology Behavior and Social Networking, 16, 4, 2013, pp. 279-284

[27] I. S. Abbasi, "Social media addiction in romantic relationships: Does user's age influence vulnerability to social media infidelity?," Personality and Individual Differences, 139, 2019, pp. 277-280

[28] V. Benson, C. Hand, and R. Hartshorne, "How compulsive use of social media affects performance: insights from the UK by purpose of use," Behaviour \& Information Technology, 38, 6, 2019, pp. 549-563 [29] W. Gao, Z. P. Liu, and J. Y. Li, "How does social presence influence SNS addiction? A belongingness theory perspective," Computers in Human Behavior, 77, 2017, pp. 347-355

[30] C. S. Andreassen, M. D. Griffiths, S. R. Gjertsen, E. Krossbakken, S. Kvam, and S. Pallesen, "The relationships between behavioral addictions and the five-factor model of personality," Journal of Behavioral Addictions, 2, 2, 2013, pp. 90-99 [31] B. Alzougool, "The impact of motives for Facebook use on Facebook addiction among ordinary users in Jordan," International Journal of Social Psychiatry, 64, 6, 2018, pp. 528-535

[32] N. Hawi and M. Samaha, "Identifying commonalities and differences in personality characteristics of Internet and social media addiction profiles: traits, self-esteem, and self-construal," Behaviour \& Information Technology, 38, 2, 2019, pp. 110-119

[33] F. Banyai et al., "Problematic Social Media Use: Results from a Large-Scale Nationally Representative Adolescent Sample," Plos One, 12, 1, 2017, [34] C. S. Andreassen, S. Pallesen, and M. D. Griffiths, "The relationship between addictive use of social media, narcissism, and self-esteem: Findings from a large national survey," Addictive Behaviors, 64, 2017, pp. $287-293$

[35] W. J. Xie and K. Karan, "Predicting Facebook addiction and state anxiety without Facebook by gender, trait anxiety, Facebook intensity, and different 
Facebook activities," Journal of Behavioral Addictions, 8, 1, 2019, pp. 79-87

[36] A. H. Busalim, M. Masrom, and W. Zakaria, "The impact of Facebook Addiction and self-esteem on students' academic performance: A multi-group analysis," Computers \& Education, 142, 2019, [37] D. Chae, H. Kim, and Y. A. Kim, "Sex Differences in the Factors Influencing Korean College Students' Addictive Tendency Toward Social Networking Sites," International Journal of Mental Health and Addiction, 16, 2, 2018, pp. 339-350 [38] S. Rothen et al., "Disentangling the role of users' preferences and impulsivity traits in problematic Facebook use," Plos One, 13, 9, 2018, [39] R. Martinez-Pecino and M. Garcia-Gavilan, "Likes and Problematic Instagram Use: The Moderating Role of Self-Esteem," Cyberpsychology Behavior and Social Networking, 22, 6, 2019, pp. 412-416

[40] I. S. Abbasi, "Social Media and Committed Relationships: What Factors Make Our Romantic Relationship Vulnerable?," Social Science Computer Review, 37, 3, 2019, pp. 425-434

[41] A. J. van Rooij, C. J. Ferguson, D. van de Mheen, and T. M. Schoenmakers, "Time To Abandon Internet Addiction? Predicting Problematic Internet, Game, And Social Media Use From Psychosocial Well-Being And Application Use," Clinical Neuropsychiatry, 14, 1, 2017, pp. 113-121

[42] J. E. Klobas, T. J. McGill, S. Moghavvemi, and T. Paramanathan, "Compulsive YouTube usage: A comparison of use motivation and personality effects," Computers in Human Behavior, 87, 2018, pp. 129-139 [43] I. Sural, M. D. Griffiths, K. Kircaburun, and E. Emirtekin, "Trait Emotional Intelligence and Problematic Social Media Use Among Adults: The Mediating Role of Social Media Use Motives," International Journal of Mental Health and Addiction, 17, 2, 2019, pp. 336-345

[44] P. K. Masur, L. Reinecke, M. Ziegele, and O. Quiring, "The interplay of intrinsic need satisfaction and Facebook specific motives in explaining addictive behavior on Facebook," Computers in Human Behavior, 39, 2014, pp. 376-386

[45] N. A. Ceballos, K. Howard, S. Dailey, S. Sharma, and T. Grimes, "Collegiate Binge Drinking and Social Media Use Among Hispanics and Non-Hispanics," Journal of Studies on Alcohol and Drugs, 79, 6, 2018, pp. 868-875

[46] A. Moreau, S. Laconi, M. Delfour, and H. Chabrol, "Psychopathological profiles of adolescent and young adult problematic Facebook users," Computers in Human Behavior, 44, 2015, pp. 64-69 [47] K. L. Chung, I. Morshidi, L. C. Yoong, and K. N. Thian, "The role of the dark tetrad and impulsivity in social media addiction: Findings from Malaysia," Personality and Individual Differences, 143, 2019, pp. 62-67

[48] Q. Li, X. H. Guo, and X. Bai, "Weekdays or weekends: Exploring the impacts of microblog posting patterns on gratification and addiction," Information \& Management, 54, 5, 2017, pp. 613-624

[49] J. H. Tang, M. C. Chen, C. Y. Yang, T. Y. Chung, and Y. A. Lee, "Personality traits, interpersonal relationships, online social support, and Facebook addiction," Telematics and Informatics, 33, 1, 2016, pp. $102-108$

[50] Y. Kanat-Maymon, L. Almog, R. Cohen, and Y. Amichai-Hamburger, "Contingent self-worth and Facebook addiction," Computers in Human Behavior, 88, 2018, pp. 227-235

[51] M. Iranmanesh, B. Foroughi, D. Nikbin, and S. S. Hyun, "Shyness, self-esteem, and loneliness as causes of FA: The moderating effect of low self-control," Current Psychology,

[52] B. Foroughi, M. Iranmanesh, D. Nikbin, and S. S. Hyun, "Are depression and social anxiety the missing link between Facebook addiction and life satisfaction? The interactive effect of needs and self-regulation," Telematics and Informatics, 43, 2019,

[53] I. Vaghefi and L. Lapointe, "When too much usage is too much: Exploring the process of it addiction," in 2014 47th Hawaii International Conference on System Sciences, 2014: IEEE, pp. 44944503

[54] S. B. Choi and M. S. Lim, "Effects of social and technology overload on psychological well-being in young South Korean adults: The mediatory role of social network service addiction," Computers in Human Behavior, 61, 2016, pp. 245-254

[55] K. Burnell and T. L. Kuther, "Predictors of Mobile Phone and Social Networking Site Dependency in Adulthood," Cyberpsychology Behavior and Social Networking, 19, 10, 2016, pp. 621-627

[56] H. M. Pontes, "Investigating the differential effects of social networking site addiction and Internet gaming disorder on psychological health," Journal of Behavioral Addictions, 6, 4, 2017, pp. 601-610

[57] H. Bata, I. Pentina, M. Tarafdar, and E. B. Pullins, "Mobile social networking and salesperson maladaptive dependence behaviors," Computers in Human Behavior, 81, 2018, pp. 235-249

[58] A. M. Aladwani and M. Almarzouq, "Understanding compulsive social media use: The premise of complementing self-conceptions mismatch with technology," Computers in Human Behavior, 60, 2016, pp. 575-581 